\title{
Astronomy adapts to sharpen up its image
}

Tony Reichhardt

\section{By correcting for atmospheric distortion, adaptive optics - despite some birth pains - gives telescopes a far clearer view of the Universe.}

[WASHINGTON] The Keck Observatory in Hawaii will gain an important new capability this week when guest astronomers start using its long-awaited, \$7.5 million adaptive optics (AO) system. A team led by Shri Kulkarni of the California Institute of Technology will be the first researchers to use the system.

Like all such systems, Keck's corrects for atmospheric turbulence by sensing how light is distorted by the shifting air above and countering the distortion with a deformable mirror. The result is an order-of-magnitude improvement in image sharpness, with resolutions down to the diffraction limit, the telescope's theoretical performance limit.

The Keck AO system saw its first light in February, and has already produced images with resolutions of 22 milliarcseconds at a wavelength of 850 nanometres, very close to the diffraction limit.

The debut of adaptive optics on the tenmetre Keck II, the world's largest single telescope, and its introduction at other observatories in coming months, mark the technology's coming of age. This follows years of promises and the recent commencement of productive science using four-metre-class telescopes. Adaptive optics is "close to being ready for prime-time extragalactic astronomy", says Chris Shelton, Keck's instrument scientist for AO.

\section{Differing approaches}

Other large instruments about to start using AO systems include the eight-metre Gemini North telescope in Hawaii - already getting impressive results with an interim system designed at the University of Hawaii - and the Multiple Mirror Telescope (MMT) in Arizona. The 6.5-metre MMT will use a secondary mirror that is itself deformable, increasing the system's sensitivity. Project managers hope to have the refurbished telescope and AO system running by next spring, according to Michael Lloyd-Hart of the University of Arizona's Steward Observatory.

In October, Steward astronomers plan to place a sensitive spectrograph behind the AO-equipped 3.5-metre telescope at the US Air Force's Starfire Optical Range in New Mexico. Unlike most adaptive optics systems built for astronomical use, which are optimized for infrared observation, Starfire's system operates at shorter visible wave- lengths. This requires more correcting elements and is more technically challenging.

The air force has pioneered the use of adaptive optics to improve visual tracking of satellites from the ground. Lloyd-Hart says that "people are not aware of the advantages yet" of combining $\mathrm{AO}$ with spectroscopy. $\mathrm{He}$ adds that the new device is "exactly the tool you need" for detecting the spectral signatures of planets orbiting other stars.

So far, only a few observatories have produced consistent results with adaptive optics, such as views of binary stars, asteroids and ring arcs around Neptune (see Nature 400, 731; 1999). The 3.6-metre CanadaFrance-Hawaii Telescope (CFHT) is one of the successful ones. Its approach, pioneered by François Roddier of the University of Hawaii, uses a 'curvature sensor' to determine how natural starlight has been refracted by the atmosphere.

All AO systems require a bright source of light near the target to calibrate atmospheric distortion at a given moment. Although stars as faint as seventeenth magnitude have been used for AO correction, many objects of astronomical interest do not have such a bright object nearby, and are effectively off-limits.

The most promising solution to this problem is to bounce a laser beacon off a known layer of the atmosphere and back to the telescope. In principle, this opens the entire sky to AO-corrected viewing. Laser guide stars have been demonstrated at several locations, including Starfire and the Lick Observatory in California.

But operational laser $\mathrm{AO}$ systems have been "desperately difficult" to develop, admits astronomer Jerry Nelson of the Uni- versity of California at Santa Cruz. Nelson is the designer of the Keck Telescope and director of a new Centre for Adaptive Optics, which last month received a $\$ 20$ million grant from the National Science Foundation.

Keck has struggled with its laser system, which Shelton says is "researchy and cranky". It will not begin routine observation until next year. The Gemini project expects to solicit proposals for its system next month.

\section{Delivering the goods}

The other main drawback of adaptive optics is that it corrects for only a tiny area of sky at a time. One proposed solution is 'atmospheric tomography', which would characterize the turbulence in different layers of the atmosphere all at once, then combine the results and use several deformable mirrors to apply the correction.

In theory, this could produce diffractionlimited observation for patches of sky as much as two arcminutes wide, compared with a few arcseconds for current AO systems. But the technique is even further in the future than reliable laser guide star systems.

The CFHT system can be operated by the observer, but more complex systems under development at Keck and elsewhere require at least two technicians. Most astronomers say the technology will not be considered truly operational until fewer people are needed to operate it.

But advocates of adaptive optics believe the systems are on the threshold of general acceptance. "It's been very, very challenging," says Nelson, but AO is about to emerge from being a "technological tour de forceinto a real scientific tool".

The new centre at Santa Cruz, involving 27 partner institutions, will carry that message to astronomers, familiarize them with the technology, and develop a general set of instruments and software tools for producing and analysing the resulting data.

Meanwhile, the cost of adaptive optics systems is coming down. One small Hawaiibased company, Laplacian Optics, reportedly has more than a dozen orders for complete, operational curvature-sensing systems of the kind being used on Gemini North. The price is just $\$ 300,000$ plus installation.

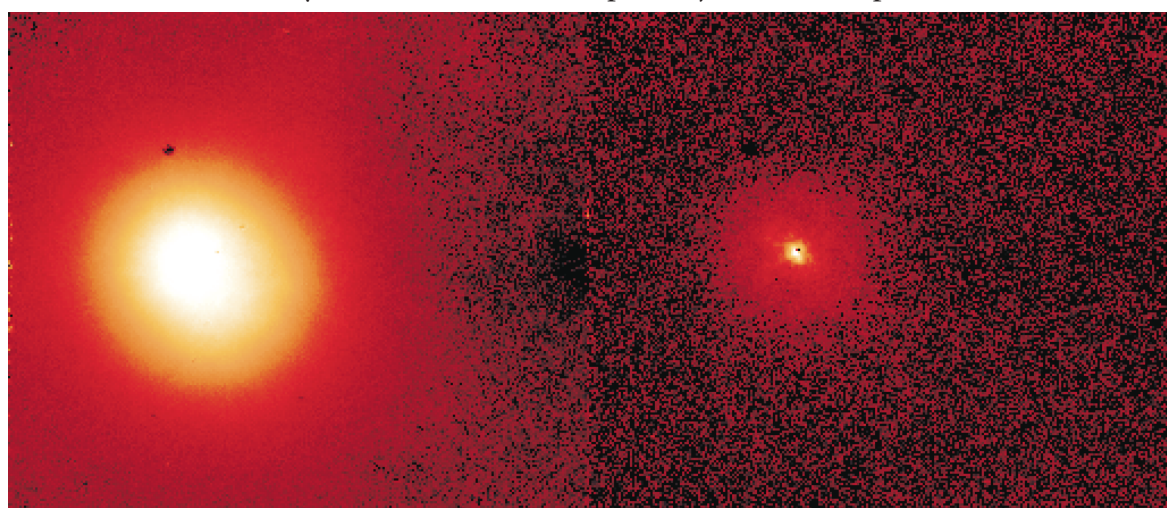

First light: a star of magnitude 5.6 viewed through Keck, with (right) and without adaptive optics. 